\title{
EL ESTUDIO DE LOS ROLES Y FUNCIONES DE LOS ORIENTADORES DE SECUNDARIA UTILIZANDO LA TÉCNICA DE LOS GRUPOS DE DISCUSIÓN
}

\section{THE STUDY OF DUTIES AND ROLES OF HIGHSCHOOL COUNSELLORS BY USING THE TECHNIQUE OF FOCUS GROUPS}

\author{
Ángel Hernando Gómez* y María del Valle Cecilia Montilla Coronado* \\ Departamento de Psicología. Universidad de Huelva
}

\begin{abstract}
RESUMEN
En el presente artículo se exponen los resultados y conclusiones de un estudio realizado, utilizando la técnica de Grupos de Discusión, sobre los roles y funciones que desempeñan los orientadores que realizan su labor en Centros de Educación Secundaria, tanto públicos como privados, de Huelva y su provincia. El objetivo perseguido era analizar la actuación profesional de los orientadores en los Institutos de Educación Secundaria de Huelva y su provincia. Dados los objetivos del estudio se decidió la utilización de la técnica del Grupo de Discusión. Participaron 34 orientadores distribuidos en cinco grupos, cuatro formados por orientadores que trabajaban en Centros Públicos y uno por orientadores de Centros Privados Concertados, que contaron entre cinco y nueve participantes. En este estudio, se presentan el proceso del análisis realizado, los resultados más destacados de la investigación, así como las conclusiones a las que se llegaron.
\end{abstract}

Entre las conclusiones a destacar encontramos:

a) La acción orientadora está excesivamente mediatizada por la "sensibilidad" del Equipo Directivo del Centro.

b) Las funciones más desarrolladas por los orientadores son las comprendidas en el asesoramiento y consulta, seguidas de orientación, evaluación y coordinación.

c) La problemática cotidiana que encuentran los orientadores en el desempeño de su trabajo es muy variada.

d) Es necesario aclarar, delimitar y concretar las funciones que deben desempeñar los orientadores.

\footnotetext{
* Hernando Gómez y Motilla Coronado, son licenciados en Psicología y Psicopedagogía y doctores por la Universidad de Huelva. Trabajan como Orientadores de Secundaria y como Profesores Asociados del área de Psicología Evolutiva y de la Educación, del Departamento de Psicología de la Universidad de Huelva. Líneas de investigación: roles y funciones de los Orientadores de Secundaria, programas de intervención en centros de Secundaria, orientación vocacional y profesional en Secundaria, prevención del maltrato de género en adolescentes. angel.hernando@dpsi.uhu.es
} 
e) A pesar de que los orientadores reconocen las ventajas de trabajar mediante un modelo de programas se ven obligados a tener que hacerlo a demanda y sometidos a la fuerte presión del día a día.

f) Debido a la poca definición de sus funciones, la realización de éstas está excesivamente influenciada por las características personales del orientador.

Palabras clave: Grupos de Discusión, orientadores, roles y funciones.

\begin{abstract}
The following paper exposes the results and conclusions of a study where the technique of Focus Groups about roles and duties of Highschool Counsellors in both private and public school of Huelva and it's province, are done.

The main goal was to analize the profesional situation of the counsellors in Huelva's highschools and province. As the objectives of the study were given beforehand, the technique used was focus groups. Thirty four counsellors were distributed in four groups; of those four of them worked in Public centres and one in a private-concerted one with a total which goes from four to nine participants. This works presents the process of anaylisis, the most representative result of the investigation and the final conclusions which are:

a. The tutorial guidance is mediated by the sensitivity of the school's head committee.

b. The most developed duties of the counsellors are those of advice and enquires, a long with guidance, assessment and coordination.

c. The assorted problems that the counsellor finds in his day by day work.

d. It is necessary to clear up, delimit and specify the duties that the counsellors have to do.

e. Although Counsellors know the advantages of working with a model of programmes they have to work demandded by daily pressures.

f. Due to the few definitions of their duties, the fulfilment of these is tremendoualy influences by the personal caracteristics of the counsellor in question.
\end{abstract}

Key words: Focus Groups, counsellors, roles and duties.

\title{
Introducción
}

Los roles y funciones de los orientadores han sido profusamente tratados en la literatura (Gibson y Mitchell,1995; Sobrado, 1996; Sanz Oro, 1999; Repetto, Ballesteros y Malik, 1999; Hiebert, 2000; Gomariz y Hernández, 2003; Hernando y Montilla, 2005). Del mismo modo, son muchas las investigaciones previas realizadas, sobre las percepciones que tienen los orientadores de Secundaria acerca de sus propias funciones. De todas ellas, sólo algunas del ámbito internacional (Hutchinson, Barrick y Groves, 1986; Tennyson, Millar, Skovholt y Williams, 1989; Partin, 1990) y nacional (Arza, 1997; Cano, 2001; Velaz de Medrano y otros, 2001; Royo, 2002; Boza, 2003) se centran específica y exclusivamente en la figura de los orientadores de Enseñanza Secundaria. Tenemos que resaltar que, en la revisión realizada de las investigaciones previas, sólo hemos encontrado dos (Álvarez Rojo y otros, 1992; Velaz de Medrano y otros, 2001) en las que se utiliza la técnica del Grupo de Discusión.

La técnica del Grupo de discusión facilita la aparición de discursos, el contraste de opiniones y posturas de los participantes en el grupo y la negociación de identidades dentro del mismo. En opinión de Krueger (1991) el grupo de discusión es un tipo de grupo especial, en relación a sus objetivos, su tamaño, composición y procedimientos. Elegimos esta técni- 
ca por considerarse un procedimiento que es muy apropiado cuando el objetivo del estudio es explicar cómo perciben los sujetos una experiencia, idea o hecho. Para Canales y Peinados (1995) se trata de una técnica de investigación social, empleada por los investigadores cualitativos, que trabaja con el habla, en ella lo que alguien dice en determinadas condiciones de enunciación se asume como punto crítico en el que lo social se reproduce y cambia, ya que en toda habla se articula el orden social y la subjetividad. El grupo de discusión, según el autor antes citado, presenta como características definitorias: el implicar a un grupo homogéneo de personas en una interacción social, tener como objetivo recoger datos cualitativos a partir de una discusión controlada y ser una estrategia cualitativa de dicha recogida, simultáneamente inductiva y naturalista. Como ventaja nos ofrece el recoger datos de la vida real en un entorno social, ser flexible, poseer una gran validez subjetiva y ofrecer resultados rápidos a costes reducidos. La distensión que se produce en la discusión grupal, y la libertad existente a la hora de manifestar opiniones, permite que sus participantes, puedan divulgar sus emociones y percepciones de las temáticas tratadas de una manera y con una profundidad difíciles de conseguir con otras técnicas de investigación.

\section{Método}

El objetivo general que pretendíamos era el conocimiento de los roles y funciones que desempeñan los orientadores y orientadoras que realizan su labor en Centros de Educación Secundaria, tanto públicos como privados, de la provincia de Huelva. Se pretende, por tanto, realizar un análisis de necesidades de la actuación profesional de los orientadores de Huelva y su provincia que nos permita ofrecer una serie de aportaciones y propuestas, a partir de un conocimiento en profundidad, y directo, derivado de las opiniones de los propios profesionales, de cual es la situación actual de la acción orientadora en los Institutos de Educación Secundaria de Huelva.

En función de los objetivos que nos planteábamos en la investigación, se llevaron a cabo cinco grupos de discusión con una muestra formada por 34 orientadores y orientadoras, tal y como se expone en la tabla $\mathrm{n}^{\mathrm{o}} 1$, que desempeñaban sus funciones en Centros de Enseñanza Secundaria de Huelva capital y provincia. Cuatro de los grupos estaban compuestos por orientadores que trabajaban en Centros públicos y uno por los que realizaban su trabajo en Centros concertados.

El procedimiento seguido comenzó con la selección de los participantes; ésta se llevó a cabo entre los orientadores en ejercicio utilizando para ello un directorio ya elaborado; aunque para la selección inicial de los participantes no tuvimos en cuenta específicamente variables diferenciales, sí que nos aseguramos que la composición de cada grupo cumpliera unas condiciones que asegurara la representación equilibrada en ellos de: género, edad, titulación, etapas educativas, experiencia docente y orientadora, situación administrativa, antigüedad de creación del Departamento y tamaño del Centro. La decisión de realizar un grupo en el que sólo intervinieran orientadores y orientadoras que trabajaban en Centros concertados fue tomada porque pensamos que, debido a las peculiares características que tienen estos Centros con respecto a aspectos organizativos, legales y de cultura profesional, este grupo nos permitiría recoger las percepciones específicas de estos profesionales, y destacarlas sin que quedaran diluidas en la generalidad de los Centros públicos. 
TABLA 1. Grupos de Discusión: componentes, duración y lugar de celebración.

\begin{tabular}{|l|c|c|l|}
\cline { 2 - 4 } \multicolumn{1}{c|}{} & Componentes & Grabación & Lugar de realización \\
\hline Grupo No 1 & 9 & 1 h. y $33^{\prime}$ & Huelva capital \\
\hline Grupo No 2 & 5 & 1 h. y $18^{\prime}$ & Cartaya \\
\hline Grupo No3 & 6 & 1 h. y $20^{\prime}$ & Huelva capital \\
\hline Grupo No 4 & 7 & 1 h. y $23^{\prime}$ & La Palma del Condado \\
\hline Grupo No5 & 7 & 1 h. y $15^{\prime}$ & Huelva capital \\
\hline
\end{tabular}

Las sesiones de los grupos de discusión fueron grabadas en cinta magnetofónica para recoger la producción literalmente y en toda su extensión. Además de la grabación de las sesiones tanto el moderador como, sobretodo, el ayudante, recogieron anotaciones de los aspectos no verbales de las intervenciones y de cualquier otro dato que consideraran que podría servirles para el posterior proceso de análisis de datos.

En el guión de presentación se exponía la temática a tratar, de manera que se pudiera establecer el contexto de las preguntas. Se realizaron preguntas abiertas a fin de facilitar las respuestas de los participantes, éstas estaban ordenadas de lo más general a lo más específico. Las preguntas matrices, utilizadas en los grupos, se recogen en la Tabla $\mathrm{n}^{\circ} 2$. Tal como se ha dicho, son preguntas abiertas, de manera que permitieran a los orientadores y orientadoras, dentro de la temática tratada, marcar la dirección de sus respuestas y responder siguiendo distintas dimensiones; de tal forma que no se determinaban ni se sugerían las respuestas, sino que lo que pretendíamos era que cada profesional contestara basándose en su propia situación personal y de acuerdo con las percepciones y opiniones que él tenía de su realidad.

TABLA 2: Guión de la entrevista para los orientadores.

\begin{tabular}{|l|}
\hline - Hablemos sobre las actividades que desarrolláis en vuestro trabajo ¿Qué opináis del trabajo que \\
lleváis a cabo en vuestros Centros y de las funciones que desempeñáis?
\end{tabular}


Para el análisis de datos, lo primero que se tuvo presente fueron los objetivos de nuestro estudio, y dadas sus características y el carácter descriptivo de éste, decidimos no seguir un modelo previo único, ni utilizar ningún programa informático de análisis de textos, ni partir de categorías rígidas previamente establecidas; sino categorizar posteriormente las respuestas registradas, tomando como referente el estudio de las cinco dimensiones en las que se centra la investigación: Asesoramiento y consulta, Orientación, Mediación, Coordinación y Evaluación, diagnóstico y seguimiento. La utilización de esta técnica nos permitía una aproximación a los objetivos de nuestro estudio que estaría mucho más centrada en la interpretación y comprensión de los discursos.

Para el análisis de datos se optó (dentro del continuo de profundidad del análisis de los Grupos de Discusión, nos moveríamos en una bipolaridad que iría desde la presentación de los datos directos en un polo hasta la interpretación en otro, pasando por las afirmaciones descriptivas) por el nivel interpretativo, que se va a apoyar en el proceso descriptivo de los discursos pero intentando apuntar hacia la comprensión de éstos. Este nivel va más allá de la utilización de las frases descriptivas, pues utilizamos las anotaciones recogidas por el moderador y el ayudante en la realización de los grupos, el clima que rodeaba las afirmaciones y comentarios realizados por los participantes y la consistencia o labilidad de estas afirmaciones. El material utilizado para el análisis de los datos fue: el guión de la entrevista, las cintas grabadas y las trascripciones de los cinco grupos, las características de los participantes en cada grupo y las hojas de anotaciones del moderador del grupo y del ayudante.

\section{Resultados}

A continuación exponemos los resultados encontrados que se presentan ordenados en base a las preguntas y dimensiones utilizadas, y dentro de éstas, organizados en base a las categorías encontradas tras el análisis de datos y la revisión de los comentarios a todas las preguntas, categorías que giran alrededor de los puntos clave o de las ideas básicas descubiertas en los grupos de discusión.

Encontramos que las funciones de asesoramiento y consulta a padres son ampliamente realizadas teniendo muchos de ellos reuniones periódicas establecidas. Como dificultad manifiestan que la asistencia de los padres a las reuniones colectivas es muy baja, aunque se detecta cada año que la figura del orientador es cada vez más conocida, aceptada y requerida por los padres. Con respecto al asesoramiento y consulta a profesores, éste se realiza con mucha asiduidad, sobre todo, a tutores de Secundaria. Se detecta un cambio, a mejor, en las percepciones que los profesores tienen de los orientadores debido a la eficacia observada. Los orientadores entienden que el asesoramiento a profesores debe realizarse de forma grupal para evitar la multiplicidad y optimizar los recursos, consideran necesario que en estas reuniones esté siempre presente un miembro del Equipo Directivo, encontramos que gran parte de los orientadores realizan este asesoramiento grupal a profesores, de temática variada, mediante reuniones semanales programadas. En relación al asesoramiento a Equipos Directivos, a pesar de que la normativa encomienda este asesoramiento a los orientadores, la falta de concreción de sus funciones, produce que el ejercicio de esta actividad dependa de la voluntariedad de los componentes del Equipo Directivo. La mayoría de los orientadores está de acuerdo en la necesidad de que el Equipo Directivo respalde y valore la acción orientadora para que ésta pueda llevarse a cabo de una forma correcta y rentable. La rela- 
ción orientador-Equipo Directivo no está siempre libre de suspicacias y en muchas ocasiones existe desconfianza mutua. Estos resultados corroboran los datos obtenidos en estudios de investigación pioneros (Hutchinson, Barrick y Groves, 1986) y otros más recientes (Tennyson, Millar, Skovholt y Williams, 1989; Hillman, 1989; Partin, 1990; Sobrado, 1997; Malik, 1997; Cano 2001; Boza, 2003), en los que se destaca la importancia de las funciones comprendidas dentro del rol de asesoramiento y consulta.

Las funciones que más realizan, dentro del rol de orientación, son las relacionadas con programas de orientación vocacional, que son llevadas a cabo por los tutores con la colaboración y asesoramiento de los orientadores. También se realizan las funciones de orientación mediante programas o sesiones estructuradas de tutoría, diseñadas por el orientador y desarrolladas por el tutor, con el grupo clase. Estos resultados confirman los encontrados en estudios clásicos (Tennyson et al., 1989; Partin, 1990; Álvarez Rojo y otros, 1992; Gibson y Mitchell, 1995) y se mantienen en la línea de aquellos otros realizados con carácter más reciente y más cercano a nuestro contexto (Sanz Oro, 1999; Cano, 2001; Boza, 2003).

La mediación es uno de los roles menos mencionados, es posible que sea debido a las dificultades que plantea la realización de las funciones englobadas en él y que pueden llegar a poner a los orientadores en situaciones comprometidas o de enfrentamiento con sus compañeros. Se realiza sobre todo entre alumnos y profesores y entre el Centro y los padres. Los orientadores se encuentran a menudo ante problemáticas que no son de su competencia $\mathrm{y}$ ante las que realizan funciones de derivación. Coinciden nuestros resultados con los de recientes investigaciones (Hillman, 1989; Giebler, 1992; Burnjan y Jackson, 2000; Boza, 2003) que también sitúan las funciones contempladas en este rol en una calificación baja; en general, todas las funciones que se refieran a aspectos de la orientación que podríamos considerar más novedosos, han obtenido, en las últimas investigaciones realizadas, bajas puntuaciones (Sanz Oro, 1999; Burnjam y Jackson, 2000; Repetto, Malik y Ballesteros, 2000; Boza, 2003).

Son muchas las funciones de coordinación que realizan los orientadores, entre las más mencionadas se encuentran las desempeñadas con los tutores de Secundaria y con instituciones externas al Centro; se destaca que existe muy poca coordinación entre los orientadores de Secundaria y los de Primaria de los Centros adscritos al Instituto. Estos resultados coinciden con varias de las investigaciones revisadas (Tennyson et al., 1989; Arza, 1997, Sanz Oro, 1999; Cano, 2001; Royo, 2002) aunque contrasta con los encontrados en otras (Giebler, 1992; Gibson y Mitchell, 1995; Boza, 2003).

Las funciones relacionadas con los roles de evaluación y diagnóstico ocupan mucho tiempo a los orientadores; no ocurre lo mismo con las funciones de seguimiento que son poco o nada realizadas. Las funciones de evaluación y diagnóstico ocupan mucho tiempo sobre todo a comienzo de curso, provocando mucha tensión entre los orientadores que se encuentran entre un gran número de alumnos que necesitan ser evaluados, y unos profesores que no actúan hasta que no se les entregan los informes. Muchos orientadores se muestran reacios a entregar informes escritos de las valoraciones realizadas, sobre todo debido al fuerte carácter estigmatizador que pueden tener si éstos no son utilizados correctamente. La realización de las ACIs, aspecto muy relacionado con la evaluación, es un punto que resulta polémico; algunos orientadores no ven en ellas ninguna panacea e incluso se niegan a remitirlas a la Inspección educativa y optan por una posición intermedia que consiste en realizarlas pero sin seguir el protocolo establecido. Con respecto al seguimiento, encontramos 
que no se realiza con ninguno de los alumnos que salen del Centro, o sólo con algún colectivo específico. Estos resultados se mantienen en la línea de los encontrados en otras investigaciones; en ellas podemos ver que las funciones relacionadas con evaluación y diagnóstico, obtienen medias o altas puntuaciones (Hillman, 1989; Watts, 1992; Gibson y Mitchell, 1995; Sanz Oro, 1999; Repetto, Malik y Ballesteros, 1998, 2000; Burnjam y Jackson, 2000); mientras que las relacionadas con actividades de seguimiento, o no las encontramos, u obtienen puntuaciones muy bajas (Sanz Oro, 1999).

Por otra parte, consideran que la normativa que regula sus funciones es muy amplia y variable, con poca claridad en la formulación y falta de transparencia para poder desarrollarla, con necesidad de interpretación que lleva a que cada uno las aplique a su manera y conveniencia.

Los orientadores que conforman los grupos manifiestan las ventajas de trabajar mediante un modelo de programas, pero la realidad es que se ven obligados a tener que hacerlo a demanda, en función de las peticiones que le son realizadas. Entre las distintas causas que explican este cambio se encuentran: la gran amplitud de la demanda, el gran número de sujetos a los que se debe atender, la variedad y amplitud de los campos de acción y la poca especificidad de sus funciones.

Así mismo, hacen referencia a las muchas dificultades, que entorpecen su acción a la hora de desempeñar las funciones que le son encomendadas. Las dificultades mencionadas más a menudo son el hecho de que los orientadores interinos se ven obligados a cambiar cada año de Centro, lo cual supone, en muchas ocasiones, el tener que volver a empezar; la carencia de tiempo para realizar la gran multiplicidad de funciones que le son asignadas; la influencia de las características personales y profesionales del orientador que le precede en el puesto, ya que si su modelo y praxis profesional no va en la misma línea, se producen roces y contradicciones; la obligatoriedad de que algunos orientadores de la concertada tengan que llevar a cabo sus funciones en más de un Centro educativo; el bajo número de profesores de Educación Especial, especialmente acuciante en la concertada, y de otros especialistas, que dificulta o impide que puedan ser llevadas a cabo muchas de las intervenciones y acciones propuestas; la historia y el bagaje profesional que tiene el orientador cuando comienza a trabajar en la enseñanza y que, en algunas ocasiones, puede llegar a dificultar una acción de orientación educativa; el gran número de programas y acciones que vienen de fuera y a los que al D.O., como cajón de sastre, se le encarga dar respuesta; la deficiente infraestructura material (recursos y dependencias) con la que cuentan la gran mayoría de los D. de Orientación; la excesiva dependencia de la "bondad" y sensibilidad que el Equipo Directivo tenga con la tarea orientadora, que puede llegar a facilitar o a bloquear de forma determinante el desempeño del trabajo del orientador, circunstancia ésta que no se da en el resto de las especialidades.

Por otra parte, también hacen alusión al tiempo que necesitan para atender adecuadamente, y de manera individualizada, a los distintos componentes de la comunidad educativa, manifestando que se ven desbordados por la falta de tiempo.

Se da la paradoja de que aunque a priori se pudiera pensar que todos los orientadores querrían tener un segundo orientador en su Centro, aquellos pocos orientadores que han pasado por esta circunstancia son los que se muestran más reacios y encuentran más inconvenientes a la hora de compartir su trabajo con otro orientador; aun así, las opiniones están divididas. 
La totalidad de los orientadores participantes se muestra partidaria y demanda la necesidad de que los Departamentos de Orientación cuenten en sus plantillas con otros especialistas, siendo uno de los más demandados la figura del Trabajador Social.

La influencia de las características personales del orientador a la hora de realizar sus funciones fue un aspecto que, de forma recurrente, apareció y se comentó en todos los grupos, ya que se considera, de forma generalizada, que la personalidad de éste, influye en gran medida como factor facilitador o bloqueador de la acción orientadora. Estos resultados concuerdan, con ligeras variaciones, tanto con los obtenidos en investigaciones lejanas a nuestro contexto (Ibrahim, Helms y Thompson, 1983; Hutchinson, Barrick y Groves, 1986; Tennyson et al, 1989; Hillman, 1989, Partin, 1990), así como con las más recientes y próximas a nuestro contexto social y geográfico (Sobrado, 1997; Arza, 1997; Sanz Oro, 1999). En nuestra opinión este hecho corrobora una vez más que no son tantos los cambios que se están produciendo en la orientación, no obstante hay que resaltar que se va observando la configuración de un cambio lento, pero progresivo, con la aparición de novedosas funciones y "roles especiales", tal y como podemos ver en algunas investigaciones previas (Sanz Oro, 1999; Burnjam y Jackson, 2000) y con el cambio en las actitudes mantenidas por los diferentes sectores educativos con respecto a la orientación.

Para finalizar, son muchos los cambios que se demandan, entre ellos: terminar con la dependencia total del Equipo Directivo; concretar sus funciones; acabar con la soledad del orientador; los orientadores de la concertada demandan de la Administración la misma atención para sus Centros que la que tienen con los públicos, y todos, solicitan más tiempo y medios materiales y personales.

\section{Conclusiones}

Entre las conclusiones a destacar encontramos que la acción orientadora está excesivamente mediatizada por la "sensibilidad" del Equipo Directivo del Centro.

Las funciones más desarrolladas por los orientadores son las comprendidas en el asesoramiento y consulta, seguidas de orientación, evaluación y coordinación; se realizan pocas funciones de mediación, siendo casi inexistentes las de seguimiento.

La problemática cotidiana que encuentran los orientadores en el desempeño de su trabajo es muy variada y los Departamentos de Orientación adolecen de los especialistas adecuados para poder dar respuesta a todas, Es necesario dotar a los Departamentos de Orientación de los especialistas adecuados y de la infraestructura material necesaria para poder dar respuesta a las muy diversas demandas que se le plantean.

Es necesario aclarar, delimitar y concretar las funciones que deben desempeñar los orientadores en general y más específicamente las de los orientadores en los Centros concertados.

A pesar de que los orientadores reconocen las ventajas de trabajar mediante un modelo de programas se ven obligados a tener que hacerlo a demanda y sometidos a la fuerte presión del día a día; y debido a la poca definición de sus funciones, la realización de éstas está excesivamente influenciada por las características personales del orientador.

Una de las principales limitaciones de nuestro trabajo tiene que ver con el uso de una sola técnica, el Grupo de Discusión, como método de recogida de información; otra estaría 
relacionada con el hecho de sólo estudiar a una de las partes, los orientadores y orientadoras, que intervienen en los procesos de orientación, no habiéndose obtenido datos de la opinión del alumnado, familias y profesorado.

Como posibles líneas futuras de investigación consideramos necesario ampliar la población a todos los orientadores y orientadoras de Secundaria de Andalucía, realizando Grupos de Discusión en todas las provincias; esta ampliación nos podría dar un conocimiento, más exhaustivo, de la realidad de los roles y funciones de los orientadores en nuestra Comunidad, para contrastar posibles diferencias entre las distintas provincias. Asimismo, es conveniente ampliar los sectores implicados en la investigación, realizando Grupos de Discusión con el profesorado, Equipos Directivos, alumnado y familias; de manera que se puedan contrastar las opiniones de todos los sectores de la comunidad educativa.

\section{Referencias Bibliográficas}

Álvarez Rojo, V. y otros (1992). La orientación institucional en Andalucía. Aportaciones para su evaluación. Sevilla. Universidad (Grupo MIDO).

Arza, N (1997). Coordenadas contextuais e érase dos/as orientadores/as no ensino medio e secundario en Galicia. Tesis doctoral no publicada. Universidad de A Coruña.

Boza, A. (2003). Roles y funciones de los orientadores de Educación Secundaria de Huelva. Tesis doctoral no publicada, Universidad de Huelva.

Burnham, P. D. y Jackson, Z.D. (2000). "School counsellor roles: discrepancies between practice and existing models". Profesional School Counseling, 4 (1), 41-52.

Cano, R. (2001). "Los Departamentos de Orientación en los institutos de Enseñanza Secundaria: De la teoría orientadora como posibilidad a la práctica psicopedagógica como realidad”. En M. Álvarez y R. Bisquerra (Coords.), Manual de Orientación y Tutoría (pp. 95-96/26). Barcelona. Praxis.

Canales, M. y Peinados, A. (1995). “Grupos de discusión”. En J. M. Delgado y J. Gutierrez (Edit.), Métodos y técnicas cualitativas de investigación en Ciencias Sociales (pp. 287-316). Madrid. Síntesis Psicología.

Gomariz, M. A. y Hernández Fernández, J. (2003). "Los Departamentos de Orientación en la región de Murcia: Orientación y Atención a la Diversidad". Revista Española de Orientación y Psicopedagogía, 14 (2), 129-147.

Giebler, S. M. (1992). Roles and functions of the elementary counselor as perceived by elementary counsellors. Master's thesis, Fort Hayes State University, Hays, KS.

Gibson, R. L. y Mitchell, M. H. (1995). Introduction to counseling and guidance (4 edición), New York. Macmillan.

Hernando, A. y Montilla, C. (2005). "El Orientador como profesional educativo en los Centros de Enseñanza Secundaria”. En J. M. Coronel y S. González (Coord.), El trabajo psicopedagógico en los Centros de Enseñanza Secundaria (pp. 147-162). Málaga. Aljibe.

Hiebert, B. (2000). "Competencies for providing quality careers services: a look at Canadian standards for career development”. Revista Española de Orientación y Psicopedagogía, 11 (19), $1-19$.

Hillman, D. K. (1989). The importance of selected aspects of the role and function of the secondary school counselor as reported by administrators, counselors and teachers. Master's thesis, Fort Hayes State University, Hays, KS. 
Hutchinson, R. L., Barrich, A. L. y Groves, M. (1986). "Functions of secondary school counselors in the public schools: Ideal and actual". School Counselor, 43, 87-91.

Ibrahim, F., Helms, B. y Thompson, D. (1983). "Counselor rol and function: An appraisal by consumers and counsellors". Personnel and Guidance Journal, June, 597-601.

Krueger, R. (1991). El grupo de discusión. Guía práctica para la investigación aplicada. Madrid: Pirámide.

Malik, B. (1996). Las tareas del orientador: su formación inicial y en ejercicio. Un estudio en países anglosajones de la Unión Europea. Tesis doctoral.

Partin, R. L. (1990). School counselor's time: A comparison of counselors' and principals' perceptions and desires. Paper presented at Cincinnati, OH. (ERIC Doc. NL'ED 316 786).

Repetto, E., Ballesteros, B. y Malik, B. (1999). "Hacia una formación de los orientadores en Europa: Estudio empírico de las áreas de competencias más relevantes". Revista Española de Orientación y Psicopedagogía, 10 (17), 149-162.

Repetto, E., Ballesteros, B. y Malik, B. (2000). Tareas y formación de los orientadores en la Unión Europea. Madrid: UNED.

Royo, F. (2002). "Desempeño profesional de los orientadores en los institutos de Educación Secundaria de Salamanca. Creación de un instrumento de valoración y autovaloración”. Revista Española de Orientación y Psicopedagógica, 13 (1), 31-50.

Sanz Oro, R. (1999). Los departamentos de orientación en Educación Secundaria: Roles y funciones. Barcelona. Cedecs.

Sobrado, L. (1996). Servicios de Orientación os centros educativos. Santiago de Compostela. Ediciones Laivento.

Tennyson, W. W., Miller, G. D., Skovholt, T. G. y Williams, R. C. (1989). "Secondary school counselors: What do they do? What is important?". School Counselor, 36, 253-259.

Vélaz de Medrano, C., Repetto, E, Blanco, A., Guillamón, J. R., Negro, A. y (2001). "El desarrollo profesional de los orientadores de Educación Secundaria. Análisis de necesidades y prospectiva". Revista de Investigación Educativa, 19 (1), 199-220.

Fecha de recepción: 06-06-2007

Fecha de revisión: 03-07-2008

Fecha de aceptación: 10-09-2008 


\title{
HABILIDADES SOCIALES Y ACOSO ESCOLAR: UN ESTUDIO EN CENTROS DE ENSEÑANZA SECUNDARIA DE MADRID
}

\author{
SOCIAL SKILLS AND SCHOOL BULLYNG: \\ A STUDY IN SECONDARY SCHOOLS IN MADRID
}

\author{
$M^{a}$ Luisa Dueñas Buey* y María Senra Varela** \\ Universidad Nacional de Educación a Distancia
}

\section{RESUMEN}

Este artículo es el resultado de un estudio de tipo descriptivo con una muestra de adolescentes de edades comprendidas entre los 13 y 14 años con la finalidad de analizar el fenómeno del acoso escolar, así como la incidencia de ciertas habilidades sociales relevantes en relación con el mismo. Se analiza el acoso escolar a tres niveles diferenciados: bajo, medio y alto en las 9 escalas que componen el instrumento utilizado. También se analizan:

- Los niveles de acoso escolar según el sexo.

- Las habilidades sociales en función del sexo y, finalmente,

- El acoso escolar en función de las habilidades sociales.

Palabras clave: acoso escolar, habilidades sociales, niveles de acoso escolar.

\begin{abstract}
The bullying phenomenon is a critically important subject in educational settings as well as in society. The studies conducted in this line show that people with tendency to aggressive behaviour are characterized by a distinguishable profile: instability, irritability, external attribution, high levels of anxiety, low self-esteem and tendency to depression.

This paper arises from a descriptive study with a sample of adolescents (age from 13 to 14). It is focused on the analysis of the bullying phenomenon and the impact of specific social skills on it. Nine scales of bullying are analysed: harassment, intimidation, threats, coercions, social boycott, social ex-

* Profesora del Departamento MIDE II de la Facultad de Educación de la UNED. Sus líneas de investigación se centran en el ámbito del diagnóstico y la intervención educativa con especial incidencia en el campo de la educación Especial. E-mail: mduenas@edu.uned.es

** Profesora del Departamento MIDE II de la Facultad de Educación de la UNED. Sus trabajos de investigación versan actualmente sobre poblaciones especiales. E-mail: msenra@edu.uned.es
\end{abstract}

